\title{
Vulval Tuberculosis: The Histomorphological Spectrum in Patients with HIV Co-Infection and AIDS
}

\author{
Nhlonzi GB ${ }^{1}$, Ramdial PK ${ }^{1 *}$, Nargan K², Lumamba KD², Pillay B ${ }^{3}$, Kuppusamy JB ${ }^{1}$, Naidoo T1 and Steyn AJC ${ }^{2,4,5}$ \\ ${ }^{1}$ Department of Anatomical Pathology, National Health Laboratory Service and University of KwaZulu-Natal, Durban, South Africa \\ ${ }^{2}$ Africa Health Research Institute, Durban, KwaZulu-Natal, South Africa \\ ${ }^{3}$ Department of Vascular/Endovascular Surgery, Nelson R Mandela School of Medicine, University of KwaZulu-Natal and Inkosi Albert Luthuli Central Hospital, Durban, \\ KwaZulu-Natal, South Africa \\ ${ }^{4}$ Department of Microbiology, School of Medicine, University of Alabama at Birmingham, Birmingham, USA \\ ${ }^{5} U A B$ Centers for AIDS Research and Free Radical Biology, University of Alabama at Birmingham, Birmingham, USA
}

\begin{abstract}
Objective: Vulval tuberculosis (TB) is reported rarely. The histomorphological spectrum and diagnostic mimicry thereof in patients with concomitant HIV infection/ AIDS is unreported to date. This study aimed to appraise the histopathologic spectrum of vulval TB in HIV co-infected patients and to identify histopathological diagnostic challenges, mimicry and pitfalls.

Methods: Ten year retrospective study that reappraised the histomorphological features of vulval TB in HIV-infected patients.

Results: The clinical descriptions of the biopsied lesions from 19 patients that form the study cohort encompassed nodules (9), ulcers (5), hypertrophy/edema (3) and abscesses (2). The main microscopic features included necrotizing and non-necrotizing granulomatous inflammation, ulceration with a zoned inflammatory response and chronic suppurative inflammation. The necrotizing component had the typical morphology of caseative necrosis or of suppuration/karyorrhexis or an admixture of both necrosis types. Vasculitis, of varied histomorphology, was present in 15 biopsies. Infective special stains were pivotal diagnostic tools. The presence of mycobacteria on Ziehl Neelsen stains (ZNs) and absence of nonmycobacterial infective agents on additional infective stains, underpinned TB diagnosis, especially in biopsies that lacked prototypical granulomatous inflammation or of infective mimickers that manifest with a granulomatous phenotype. ZNs also confirmed mycobacteria in vasculitic foci. The absence of mycobacteria on ZNs facilitated the diagnosis of tuberculids.

Conclusion: Lesions with a common clinical appearance had heterogeneous histomorphological features, while lesions with common histopathological features demonstrated divergent clinical morphology. Infective, especially Ziehl Neelsen, stains are pivotal in the histopathological work-up of infective/inflammatory vulval biopsies. It is possible that the rarely reported anergic mononuclear or abscessing features, pseudotumoral spindle cell reactions, ulcers with a zoned inflammatory response and the presence of vasculitis of varied type, are clues to the HIV-TB tissue partnership. Increased clinicopathological investigation of and reporting on vulval TB in the HIVIAIDS afflicted population is pivotal to ascertain this.
\end{abstract}

Keywords: Tuberculosis; HIV; AIDS; Vulva; Ulcer; Granuloma; Vasculitis

\section{Introduction}

Mycobacterium tuberculosis (MTb) infection is the second leading cause of death by an infectious disease and the foremost cause of death in the context of acquired immunodeficiency syndrome (AIDS) globally. The Human Immunodeficiency Virus (HIV) and MTb coinfective partnership is an emerging pandemic in resource-constrained developing and developed nations [1]. The 2013 World Health Organization (WHO) report informed that $14.8 \%$ ( 0.8 million) of the 5.4 million new cases of tuberculosis (TB) were extrapulmonary in location [2]. Although extrapulmonary TB is associated with significant morbidity and long-term complications, sequelae and disabilities, it does not receive specific focus in global TB control programmes $[2,3]$.

Urogenital TB is the second most common form of TB in countries with severe pandemics and the third most common in low TB incidence regions [4]. Female genital tract (FGT) TB, first described in 1744, accounts for $0.2 \%$ to $2 \%$ of gynecological diseases. It is usually asymptomatic and therefore often under-diagnosed and overlooked [5]. The fallopian tubes and endometrium are most commonly involved. The ovaries and cervix are less commonly affected but vulval TB, first described in 1881, is rare, accounting for $<1 \%$ of FGT cases [6]. When symptomatic, vulval TB is characterized by a range of clinical manifestations and morphology [611], but the histopathological spectrum thereof is poorly documented. This may be a function of the rarity of vulval TB. The clinicopathological profile of FGT TB and HIV co-infection, in general, is reported even less frequently and the histopathological spectrum in the vulval setting remains undescribed, to date [12]. Because of the burden of TB and the TB/HIV co-morbid partnership worldwide [1], it is pertinent that not only the microscopic morphological familiarity of TB in this location, but also heightened recognition of overlapping features with other vulval infections [13-15], are recognized, for accurate diagnosis and effective patient management.

This study was therefore undertaken to assess the histomorphological spectrum of vulval TB in patients co-infected with $\mathrm{HIV}$, and to highlight the microscopic pitfalls and mimicry. In so doing the pivotal diagnostic role of the histopathologist in the HIV-associated $\mathrm{TB}$ syndemic, is addressed, including the challenges related to

*Corresponding author: PK Ramdial, Department of Anatomical Pathology, Level 3, Laboratory Building, Inkosi Albert Luthuli Central Hospital, 800 Vusi Mzimela Road, Mayville, 4058, KwaZulu-Natal, South Africa; Tel: +27 (0)31 2402693; + 27 (0)31 2402610; E-mail: ramdial@ukzn.ac.za; ramdialpk@gmail.com

Received July 20, 2017; Accepted August 03, 2017; Published August 10, 2017

Citation: Nhlonzi GB, Ramdial PK, Nargan K, Lumamba KD, Pillay B, et al. (2017) Vulval Tuberculosis: The Histomorphological Spectrum in Patients with HIV CoInfection and AIDS. J AIDS Clin Res 8: 719. doi: 10.4172/2155-6113.1000719

Copyright: (c) 2017 Nhlonzi GB, et al. This is an open-access article distributed under the terms of the Creative Commons Attribution License, which permits unrestricted use, distribution, and reproduction in any medium, provided the original author and source are credited. 
historical histopathological classification approaches and the changing histopathologic profile of TB in the study context.

\section{Materials and Methods}

This is a retrospective study that involved the retrieval and reevaluation of all vulval biopsies diagnosed and coded, using the SNOMED word and code search system, as 'tuberculosis' from 1 January 2006 to 31 December 2015. The study was approved by the Biomedical Research Ethics Committee of the University of KwaZuluNatal (Study number: BCA535/16). Departmental archival slides or recut and stained sections from archival blocks were retrieved and appraised. The following stains were reviewed: hematoxylin and eosin, Ziehl Neelsen, periodic acid Schiff, Giemsa, Warthin Starry and Gomori Grocott methenamine silver. The results of those biopsies that were subjected to mycobacterial, T. pallidum or K. granulomatis polymerase chain reaction (PCR) investigation for diagnostic purposes, were also reviewed. The following clinical information was extracted from departmental records: patient age, past and current clinical history, systemic illness, the results of clinical investigations (HIV, CD4 counts, Wasserman reaction, Treponema pallidum hemagglutination, Mantoux, chest radiograph), morphology of vulval lesions, clinical differential diagnosis and drug history especially anti-TB and highly active anti-retroviral therapy (HAART)]. Biopsies from patients with unknown or negative HIV status were excluded from the study. The histopathological features that were evaluated included the architectural disease pattern and the nature of the cytomorphological responses. The acid fast bacillary quantification on Ziehl Neelsen stains, using a 40X objective and $4 \mathrm{X}$ eye-piece was: negative/non-bacillary (no AFBs/10 high power fields), paucibacillary (1-3 AFBs/10 high power fields) and multibacillary ( $>3 \mathrm{AFBs} / 10$ high power fields).

The mycobacterial PCR investigations were undertaken as per local modifications of international methods $[16,17]$. Briefly, genomic DNA was extracted from formalin-fixed paraffin-embedded tissue using the QIAamp DNA formalin-fixed tissue kit (Qiagen, Hilden, Germany) according to the manufacturer's protocol. Following quantification of the extracted DNA, DNA quality was assessed by PCR amplification for a 309 bp segment of $\beta$-actin [16]. MTb complex identification was accomplished by PCR targeting of a 220 bp segment of IS6110 [17] using outer primers (O1:CGGGACCACCCGCGGCAAAGCCCGCAG GAC;O2:CATCGTGGAAGCGACCCGCCAGCCCAGGAT). Samples that were negative following agarose gel electrophoresis were then subjected to nested PCR targeting a 123 bp segment using inner primers (I1:CCTGCGAGCGTAGGCGTCGG; I2: CTCGTCCAGCGCCGCTTCGG). PCR amplification was performed on a CFX96 Real-time PCR system (BIO-RAD, CA, USA).

\section{Results}

\section{Summary of clinical findings}

28 vulval biopsies from 28 patients with TB were confirmed in the study period. Of these, 19 patients were HIV seropositive, their archival tissue and records were available for re-appraisal and they form the study cohort. The age range of patients was 21 to $47($ mean=33.4) years. While 11 patients presented with a discrete vulval mass, variably labeled as vulval abscess or nodule, 5 presented with vulval ulcers and 3 with vulval hypertrophy, edema and hyperpigmentation clinically.

\begin{tabular}{|c|c|c|c|c|c|c|c|c|c|}
\hline No & $A / R$ & CD4 & Site & P/History & ARV & Clinical & VI & Histopathol & AFB \\
\hline \multicolumn{10}{|c|}{ Clinical: Nodule $(n=9)$} \\
\hline 1 & $32 / \mathrm{A}$ & 128 & $\mathrm{Ve}$ & Nil & Nil & Sy & $\mathrm{CM}$ & $\mathrm{GT} / \mathrm{LC} / \mathrm{PC} / \mathrm{HC}$ & $\mathrm{MB}$ \\
\hline 2 & $46 / \mathrm{A}$ & 414 & MP & STI & Nil & STI & Nil & ST/NGI/NNGI & PB \\
\hline 3 & $32 / \mathrm{A}$ & 42 & LMi & TOTB & Nil & STI & Nil & NGI/NGI-NPs & MB \\
\hline 4 & $33 / \mathrm{A}$ & 155 & LMa & RTB & Nil & STI/TB & $\mathrm{CL}$ & IPT: SC/MICs & $\mathrm{MB}$ \\
\hline 5 & $47 / \mathrm{A}$ & 214 & LMa & ETB & Nil & $\mathrm{Ca}$ & IL & $\mathrm{NNGI/NGI}$ & PB \\
\hline 6 & $30 / \mathrm{A}$ & 22 & LMa & Nil & Nil & STI & KS & NNG1/NGI-NPs & MB \\
\hline 7 & $25 / \mathrm{A}$ & 261 & $\mathrm{Ve}$ & ITB & Nil & STI & nil & NNGI/NGI & PB \\
\hline 8 & $26 / \mathrm{A}$ & 108 & LMa & PTB & 2 & LGV & Nil & $\mathrm{NGI} / / N N G I$ & PB \\
\hline 9 & $22 / \mathrm{A}$ & 104 & LMa & PTB & Nil & $\mathrm{Ly} / \mathrm{Ca}$ & $\mathrm{IL}$ & $\mathrm{NGI} / / \mathrm{NNGI}$ & MB \\
\hline \multicolumn{10}{|c|}{ Clinical: Ulcers $(n=5)$} \\
\hline 10 & $31 / \mathrm{A}$ & 19 & LMa & PTB & Nil & $\mathrm{Ca} / \mathrm{TB}$ & $\mathrm{CL}$ & ZU & $\mathrm{MB}$ \\
\hline 11 & $21 / \mathrm{A}$ & 58 & $\mathrm{Ve}$ & PTB & Nil & Sy & Nil & ZU & MB \\
\hline 12 & $44 / \mathrm{A}$ & 4 & $\mathrm{Ve}$ & PTB & Nil & $\mathrm{Gl}$ & $\mathrm{CL}$ & ZU/NGI-NPs & $\mathrm{MB}$ \\
\hline 13 & $33 / \mathrm{A}$ & 44 & LMa & PTB & Nil & STI & Nil & ZU & MB \\
\hline 14 & $44 / \mathrm{A}$ & 159 & MP & TOTВ & Nil & HSV & Nil & ZU (PNT) & Nil \\
\hline \multicolumn{10}{|c|}{ Clinical: Hypertrophy/edema/induration $(n=3)$} \\
\hline 15 & $41 / \mathrm{A}$ & 314 & LMa & PTB & 3 & $\mathrm{Ca}$ & $\mathrm{CL}$ & NNGI (LS) & Nil \\
\hline 16 & $31 / 1$ & 442 & LMi & PTB & 24 & $\mathrm{Ca}$ & $\mathrm{IL}$ & NNGI (LS) & Nil \\
\hline 17 & $47 / \mathrm{A}$ & 356 & LMa & PTB & 14 & STI & $\mathrm{IL}$ & NNGI/NGI & PB \\
\hline \multicolumn{10}{|c|}{ Clinical: Abscess $(n=2)$} \\
\hline 18 & $27 / \mathrm{A}$ & 152 & LMa & ITB & 1 & Abscess & Nil & CSI & MB \\
\hline 19 & $22 / \mathrm{A}$ & 32 & $\mathrm{Ve}$ & TOTB & Nil & Abscess & Nil & CSI & $\mathrm{MB}$ \\
\hline
\end{tabular}

A: Black African; AFB: Acid Fast Bacilli; A/R: Age/Race; ARV: Duration of Antiretroviral Therapy in Months; Ca: Carcinoma; CD4: CD4 counts per cubic milliliters; CL: cervical (neck) tuberculous lymphadenopathy; Clinical: Clinical Diagnosis/Differential Diagnosis; CM: Cryptococcal Meningitis; CSI: Chronic Suppurative Inflammation; ETB: Endometrial Tuberculosis; GI: Granuloma Inguinale; GT: Granulation Tissue; HC: Histiocyte; Histopathol: Main Histopathological Features; HSV: Herpes Simplex Virus Ulcer; IL: Inguinal Tuberculous Lymphadenopathy; I:Indian; IPT: Inflammatory Pseudotumor; ITB: Intestinal Tuberculosis; KS: Kaposi Sarcoma; LC: Lymphocyte; LGV: Lymphogranuloma Venereum; LMa: Labium Majus; LMi: Labium Minus; LS: lichen scrofulosorum; Ly: Lymphoma; MB: Multibacillary; MICs: Mononuclear Inflammatory Cells; MP: Mons Pubis; NGI: necrotizing granulomatous inflammation; NNGI: Non- Necrotizing Granulomatous Inflammation; No: Number; NPs: Neutrophils; PB: Paucibacillary; PC: Plasma Cells; P/H: Past History; PNT: Papulonecrotic Tuberculid; PTB: Pulmonary Tuberculosis; RTB: Renal Tuberculosis; SC: Spindle Cells; ST: Sinus Tract; STI: Sexually Transmitted Infection; Sy: Syphilis; TB: Tuberculosis; TOTB: Tubo-Ovarian Tuberculosis; VI: Visceral Illness; Ve: Vulval Vestibule; ZU: zoned ulcer

Table 1: Summary of clinicopathological features. 
Five patients were on HAART, but in $14 / 19$ patients, the vulval disease was the sentinel of HIV infection. TB was a clinical suspicion in $2 / 19$ patients; however none were treated for TB at the time of presentation. Fifteen patients had prior visceral TB for which they were treated but the vulval disease served as the sentinel of current active TB. Based on the vulval biopsy diagnosis, fine needle aspiration cytology of neck (4) and inguinal (4) lymph nodes was undertaken that revealed active TB. One, 2, 4 and 9 patients each had been treated for prior renal, intestinal, genital and pulmonary TB, respectively (Table 1 ).

\section{Histopathological features and relevant clinical correlation}

Microscopically, biopsies from clinically similar morphological lesions demonstrated cytoarchitectural heterogeneity and variability in lesional mycobacterial density. Clinical nodular lesions $(n=9)$ : Granulomatous inflammation was noted in 7/9 biopsies (Figures 1-3) and one each was either anergic (Figures 4A-4C) or pseudotumoral (Figures 4D and 4E) in type. All of 7 biopsies with granulomas had necrotizing (NGI) and non-necrotizing (NNGI) granulomatous inflammatory lesions (Figures 1-3). The necrotizing component was either caseative (Figures 1A and 1B), suppurative and karyorrhectic (Figure 1C) or combined suppurative and caseative (patients 3 and 5) (Figure 1D) in type. The acid fast bacillary load was variable. All granulomas with a suppurative component and some with a caseative component were multibacillary (Figures $1 \mathrm{E}$ and $1 \mathrm{~F}$ ), while some caseative foci were paucibacillary (Figure 1G) (patients 2 and 4). Vascular alterations, including vasculitis of varied microscopic morphology and stage, fibroplasia, mural edema and diapedesis, were present in all biopsies and endarteritis obliterans in 3/7 (Figures 2A-2E and $2 \mathrm{G}$ ). Leucocytoclastic (Figures $2 \mathrm{~A}$ and $2 \mathrm{~B}$ ), lymphocytic (Figure
2C) and granulomatous (Figures $2 \mathrm{D}$ and $2 \mathrm{E}$ ) vasculitis was present in $7 / 7,5 / 7$ and $2 / 7$ biopsies, respectively. Acid fast bacilli (Figure $2 \mathrm{~F}$ ) were identified within the vasculature in $2 / 7$ vessels afflicted by leucocytoclastic vasculitis. In addition, endarteritis obliterans (Figure $2 \mathrm{G}$ ) was noted in $3 / 7$ biopsies. Three of the biopsies had additional features that mimicked syphilis (Figure 3A), including variable epidermal psoriasiform and atrophic reaction patterns, superficial dermal non-necrotizing granulomas, vasculitis and a dense plasma cell infiltrate. The absence of spirochetes on Warthin Starry silver staining and presence of acid fast bacilli (Figure 3B) confirmed TB. Two of 7 biopsies had additional features that mimicked granuloma inguinale (Figure 3C), including dense inflammatory granulation tissue, rich in plasma cells, neutrophilic microabscesses and scattered foamy histiocytes. The absence of Donovan bodies on silver staining and presence of acid fast bacilli (Figure 3D) facilitated the diagnosis of TB.

The biopsy from patient 1 had an anergic inflammatory response (Figures $4 \mathrm{~A}-4 \mathrm{C}$ ) that displayed a predominant non-granulomatous, mainly mononuclear, inflammatory cell response (Figure 4A), lymphocytic vasculitis (Figure 4B) and a multibacillary mycobacterial profile (Figure $4 \mathrm{C}$ ). One biopsy had an inflammatory pseudotumoral response (Figures 4D and 4E) with a dominant CD68-positive, spindled histiocytic composition (Figure 4D), that was teeming with acid fast bacilli (Figure 4E). Ulceration of 6/9 and fissuring of $1 / 9$ nodular lesions with replacement by thin granulation tissue containing mixed inflammatory cells were evident. The CD4 counts were not reflective of specific histomorphological presentations.

Clinical ulcers $(\mathrm{n}=5)$ : All ulcerative lesions demonstrated an organized zonal inflammatory response (Figure 5A). Multiple acid fast bacilli were present in extracellular and intracellular neutrophilic and

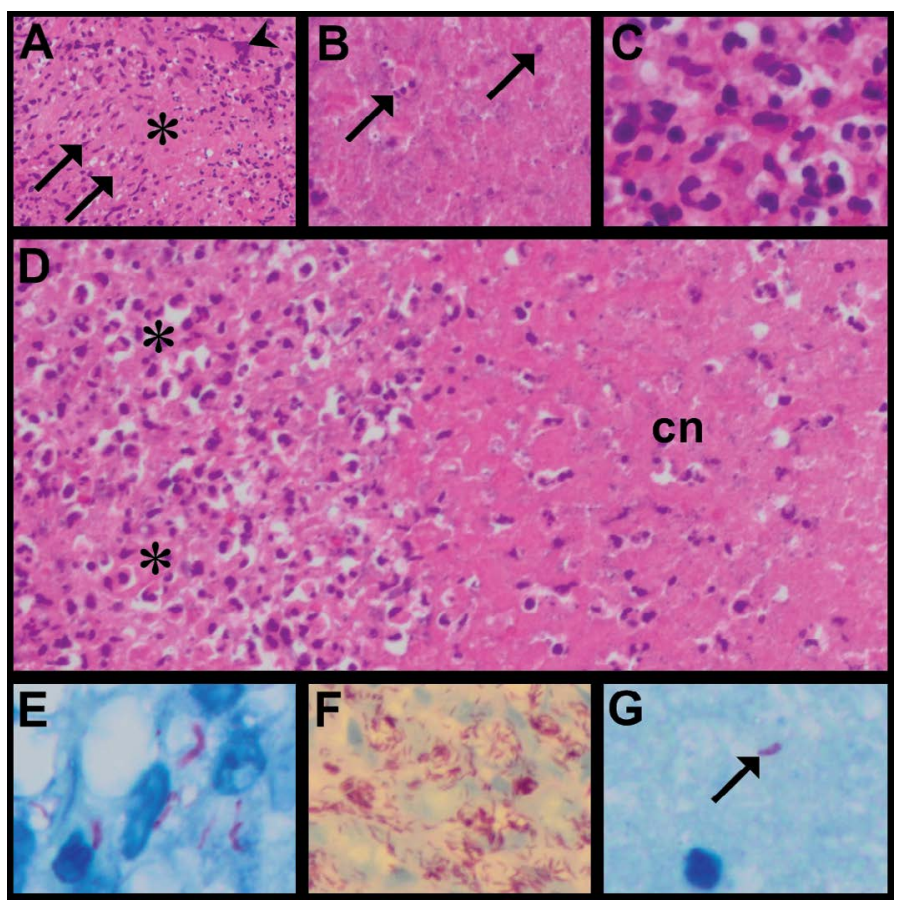

Figure 1: Microscopic features of clinical nodular lesions I: Granulomas (A): Sections of necrotizing granulomas composed of epithelioid histiocytes (arrows), Langhans giant cells (arrowhead) and caseative necrosis (asterisk) (hematoxylin and eosin). (B): High power depiction of caseative necrosis composed of eosinophilic granular material and minimal karyorrhectic debris (arrows) (hematoxylin and eosin). (C): Granuloma with suppurative inflammation and karyorrhexis (hematoxylin and eosin). (D): Admixture of caseative (cn) and suppurative (asterisks) necrosis within granuloma (hematoxylin and eosin). Myriad acid fast bacilli in suppurative (E) and caseative (F) necrotising components (Ziehl Neelsen). Focal organisms in caseative necrosis (G) (Ziehl Neelsen). 
Citation: Nhlonzi GB, Ramdial PK, Nargan K, Lumamba KD, Pillay B, et al. (2017) Vulval Tuberculosis: The Histomorphological Spectrum in Patients with HIV Co-Infection and AIDS. J AIDS Clin Res 8: 719. doi: 10.4172/2155-6113.1000719

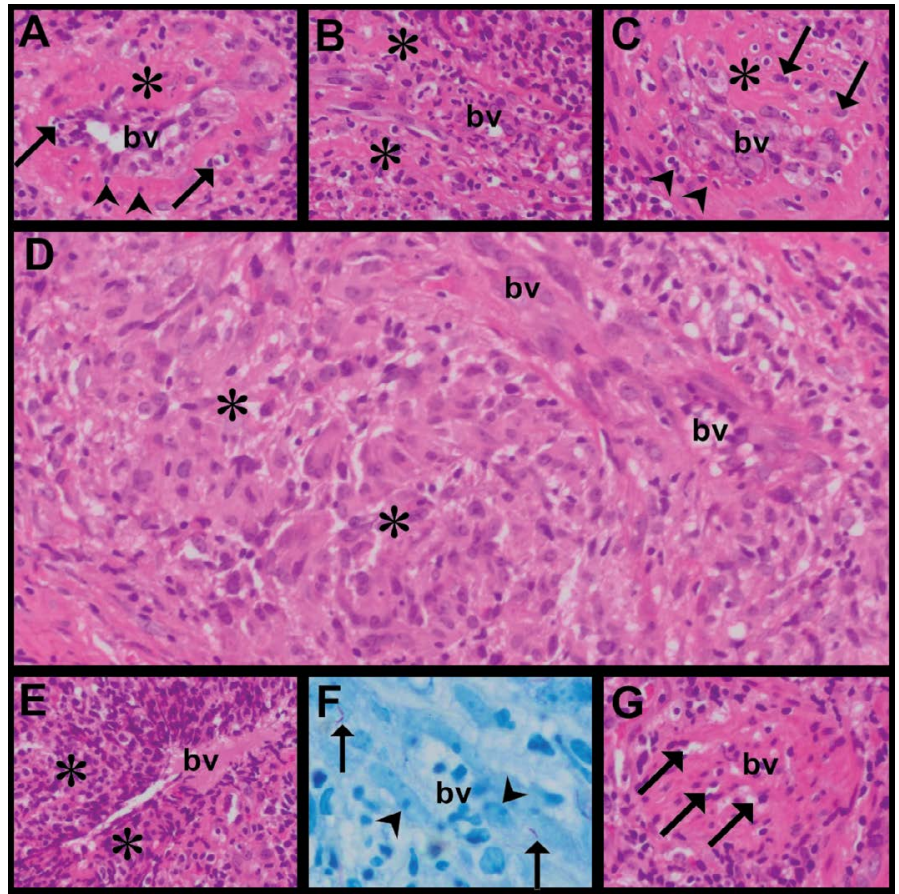

Figure 2: Microscopic features of clinical nodular lesions II: Vascular reactions. Inflamed blood vessel demonstrating leucocytoclastic vasculitis in cross (A) and longitudinal sections (B) with prominent neutrophils (A, arrows), cellular debris (A, arrowheads) and fibrinoid necrosis (A, B: asterisks) in vessel wall (hematoxylin and eosin). (C): Vessel destruction by lymphocytic vasculitis with lymphocytes (arrowheads) and fibrinoid necrosis (asterisk) in vessel wall (hematoxylin and eosin) Granulomatous vasculitis characterized by an organized granulomatous reaction ( $D$, asterisks) and dominant histiocytic infiltrate (E, asterisks) (hematoxylin and eosin). Acid fast bacilli ( $F$, arrows) in vessel (arrowheads=endothelial cells) with leucocytoclastic vasculitis (Ziehl Neelsen). Endarteritis obliterans of a small muscular artery (G) with multiple recanalized lumina (arrows) (hematoxylin and eosin); (bv=blood vessel).

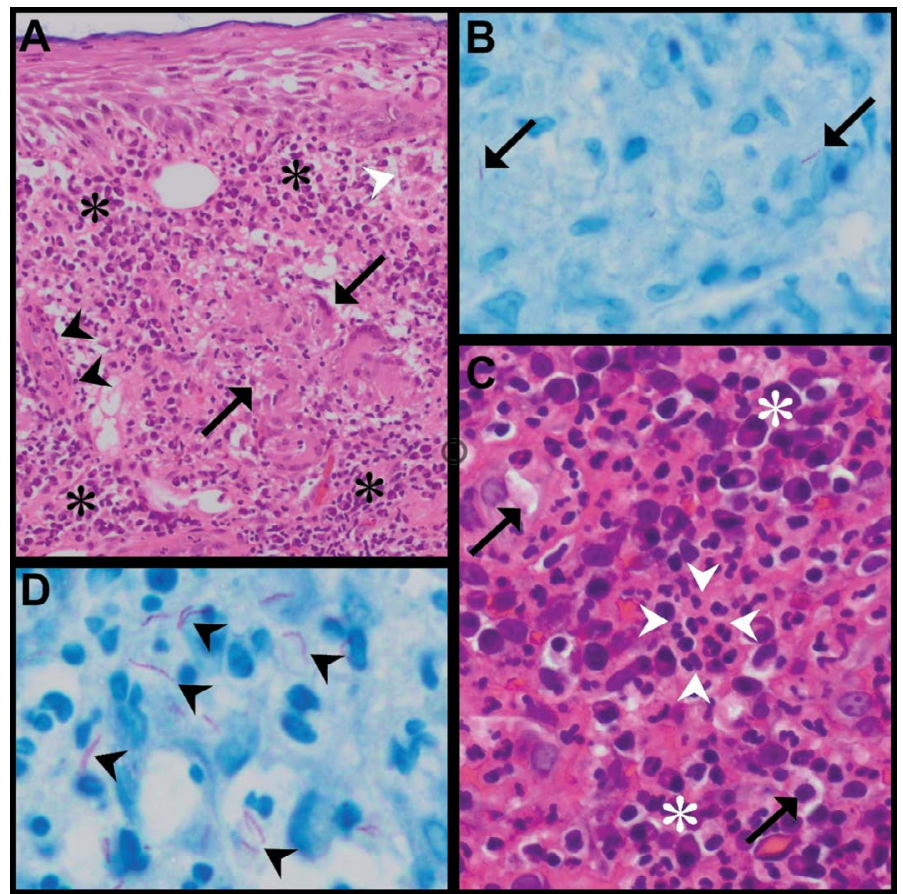

Figure 3: Microscopic features of clinical nodular lesions III: Mimickers. Non-necrotizing granulomas (A, arrows), some with a microgranulomatous appearance (white arrowhead), leucocytoclastic vasculitis (black arrowheads) and a dense plasma cell infiltrate (asterisks) superficially located in the dermis, mimicking syphilis (hematoxylin and eosin). (B): Acid fast bacilli (arrows) in non-necrotizing granuloma (Ziehl Neelsen). Dense inflammatory granulation tissue response (C), rich in plasma cells (asterisks), neutrophilic microabscess (arrowheads) and scattered foamy histiocytes (arrows) mimicking granuloma inguinale (hematoxylin and eosin) but acid fast bacilli (D, arrowheads) identified (Ziehl Neelsen). 
Citation: Nhlonzi GB, Ramdial PK, Nargan K, Lumamba KD, Pillay B, et al. (2017) Vulval Tuberculosis: The Histomorphological Spectrum in Patients with HIV Co-Infection and AIDS. J AIDS Clin Res 8: 719. doi: 10.4172/2155-6113.1000719

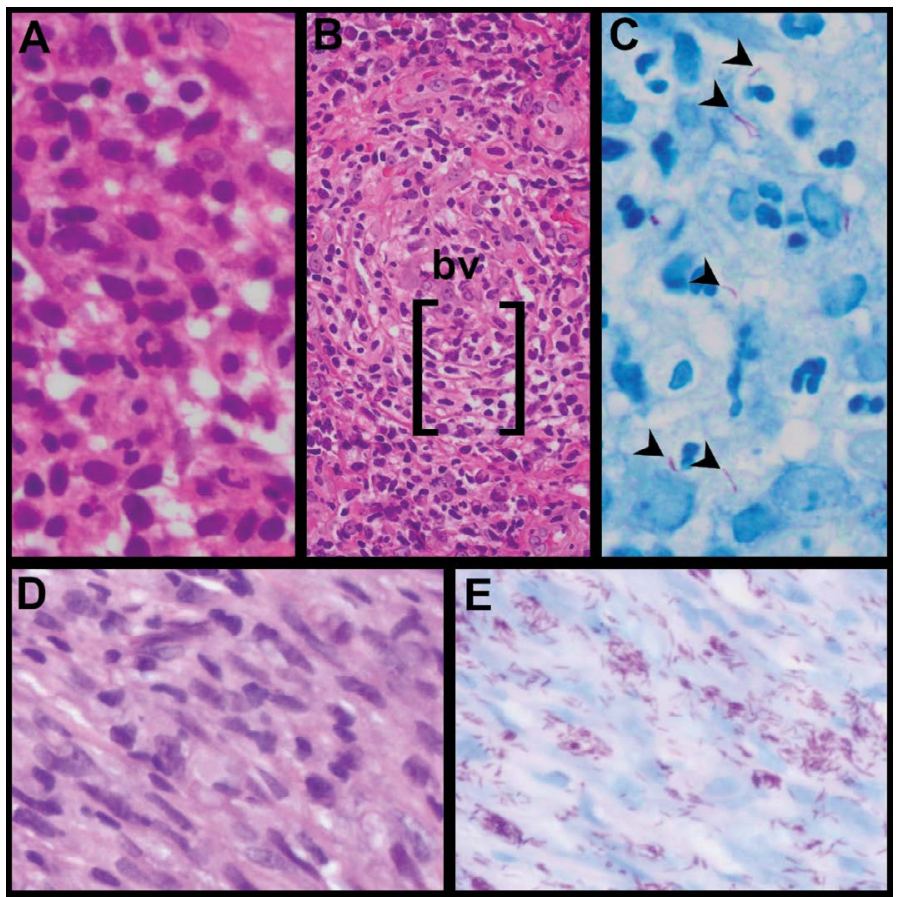

Figure 4: Microscopic features of clinical nodular lesions (IV): Anergic and pseudotumoral responses. Non-granulomatous, predominantly mononuclear anergic inflammatory response (A) with lymphocytes, histiocytes and scattered neutrophils, lymphocytic vasculitis (B) with blood vessel (bv) wall infiltration mainly by lymphocytes [square bracket] (hematoxylin and eosin). Acid fast bacilli (C, arrowheads) in inflammatory component (Ziehl Neelsen). Compact spindle cells in pseudotumoral response (D) (hematoxylin and eosin) teeming with acid fast bacilli (E) (Ziehl Neelsen).

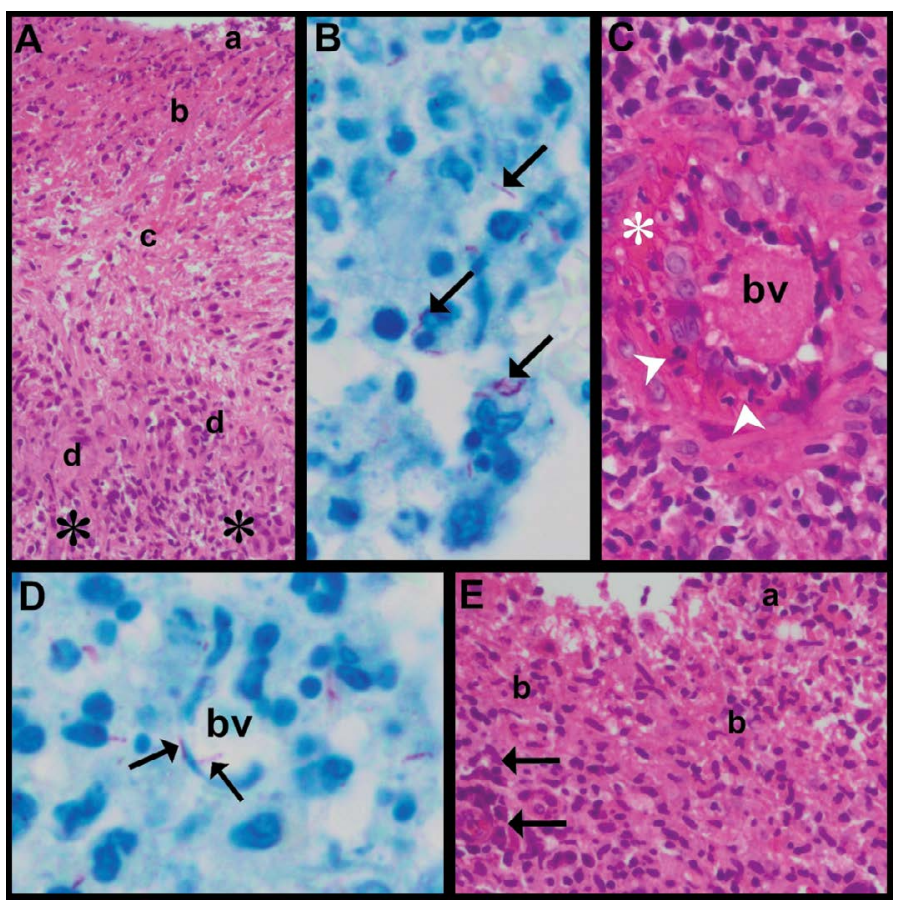

Figure 5: Microscopic features of clinical ulcerative lesions: Zoned inflammatory response (A) composed of a superficial exudative zone of neutrophils and karyorrhectic debris (a), a second intensely eosinophilic and hypocellular fibrinoid zone (b), a third hypocellular vascularized zone (c), and a deep granulomatous inflammatory zone(d) on inflamed granulation tissue (asterisks) (hematoxylin and eosin). Multiple acid fast bacilli in exudative layer (B, arrows) (Ziehl Neelsen). Leucocytoclastic vasculitis (C) with fibrinoid necrosis (asterisk) of blood vessel (bv) wall with neutrophils and nuclear debris (arrowheads) (hematoxylin and eosin). Acid fast bacilli in inflamed vessel wall (D, arrows) (Ziehl Neelsen). Papulonecrotic tuberculid with zoned inflammatory response including exudative (a) and granulomatous (b) zones and adjacent vasculitis (arrows) (hematoxylin and eosin). 
Citation: Nhlonzi GB, Ramdial PK, Nargan K, Lumamba KD, Pillay B, et al. (2017) Vulval Tuberculosis: The Histomorphological Spectrum in Patients with HIV Co-Infection and AIDS. J AIDS Clin Res 8: 719. doi: 10.4172/2155-6113.1000719

Page 6 of 10

histiocytic compartments, but mainly in the superficial, exudative zone (Figure 5B). A transitional zone of inflammatory granulation tissue merged with surrounding residual vulval fibroconnective tissue. Within the dermal granulation tissue, fibrin thrombi, vasculitis and endarteritis obliterans were present in all biopsies. One biopsy demonstrated lymphocytic vasculitis and 3 demonstrated leuocytoclastic vasculitis (Figure 5C). Acid fast bacilli were identified in leucocytoclastic vasculitic foci of 2 biopsies (Figure 5D). The CD4 count was low in all patients with ulcerative lesions, including in patient 14 , in whom the ulcer had a similar zonal inflammatory pattern (Figure 5E) but was non-bacillary, in keeping with papulonecrotic tuberculid. Clinical correlation confirmed disseminated extragenital pulmonary TB and disseminated cutaneous papulopustular and papulonecrotic lesions on the face, earlobes, trunk and limbs of patient 14 that responded dramatically to anti-tuberculous treatment. Clinical hypertrophic/edematous lesions $(n=3)$ : Biopsies from $2 / 3$ patients (patients 15 and 16 ) with vulval edema demonstrated non-necrotizing microgranulomas in peri-eccrine (Figure 6A), perifollicular and perivascular dermal locations and associated dilated lymphatic (Figure 6B) and blood (Figure 6C) vessels. Acid fast bacilli were not identified. Clinical correlation confirmed a disseminated papular eruption in both patients that supported a diagnosis of lichen scrofulosorum. The biopsy from patient 17 demonstrated NGI and NNGI and dilated lymphatic channels. Acid fast bacilli were identified within the NNGI. Patients 15, 16 and 17 had inguinal lymphadenopathy; fine needle aspiration cytology confirmed active TB. The CD4 counts were relatively higher in these patients. Clinical abscesses $(n=2)$ : Both biopsies with a clinical abscessing morphology (patients 18 and 19) demonstrated necrotizing, chronic suppurative inflammation in granulation tissue (Figures 6D and 6E), associated vasculitis and myriad acid fast bacilli, including in inflamed vessels (Figure 6F). The CD4 count was low in both patients (Table 1).
Additional infective stains did not identify further co-morbid infective pathology. MTb DNA was confirmed in 16/19 biopsies. A negative yield was confirmed in 3 biopsies of tuberculids (patients 14, 15 and 16). The T. pallidum and K. granulomatis PCR investigations were negative in 3 and 2 biopsies that mimicked syphilis and granuloma inguinale, respectively.

\section{Discussion}

$\mathrm{TB}$, an ancient infection that has plagued mankind through many millennia, remains a major health problem. It is the cause of higher morbidity and mortality rates than any other infection globally but is eminently treatable if diagnosed timeously and correctly. Notwithstanding this, 2015 WHO statistics have ranked TB higher than HIV infection/AIDS as one of the foremost causes of death from an infectious disease. In addition to approximately 1.4 million deaths caused by TB in 2015, there were a further 0.4 million deaths from TB in patients co-infected with HIV. TB-HIV co-infection was highest in WHO African region countries, exceeding $50 \%$ in parts of Southern Africa. HIV-infected patients have a 20 to 37 times risk of developing TB than those that are HIV uninfected [18]. The HIV immunosuppression enables MTb infection and the progression to active TB [19-21]. Of 10.4 million new TB cases reported in 2015, 3.5 million occurred amongst women. TB is amongst the top 5 killers of adult women, 20-59 years of age and accounts of one third of all AIDS-associated deaths in adult women. Germane to the South African context of this study, $90 \%$ of HIV-related TB deaths were in women in Africa [21].

The histomorphological heterogeneity of pulmonary and extrapulmonary TB is recognized [22-25]. Although several workers [22-24] have described the lesional features of treated and untreated pulmonary TB, it was only in 1987 that Ridley and Ridley presented

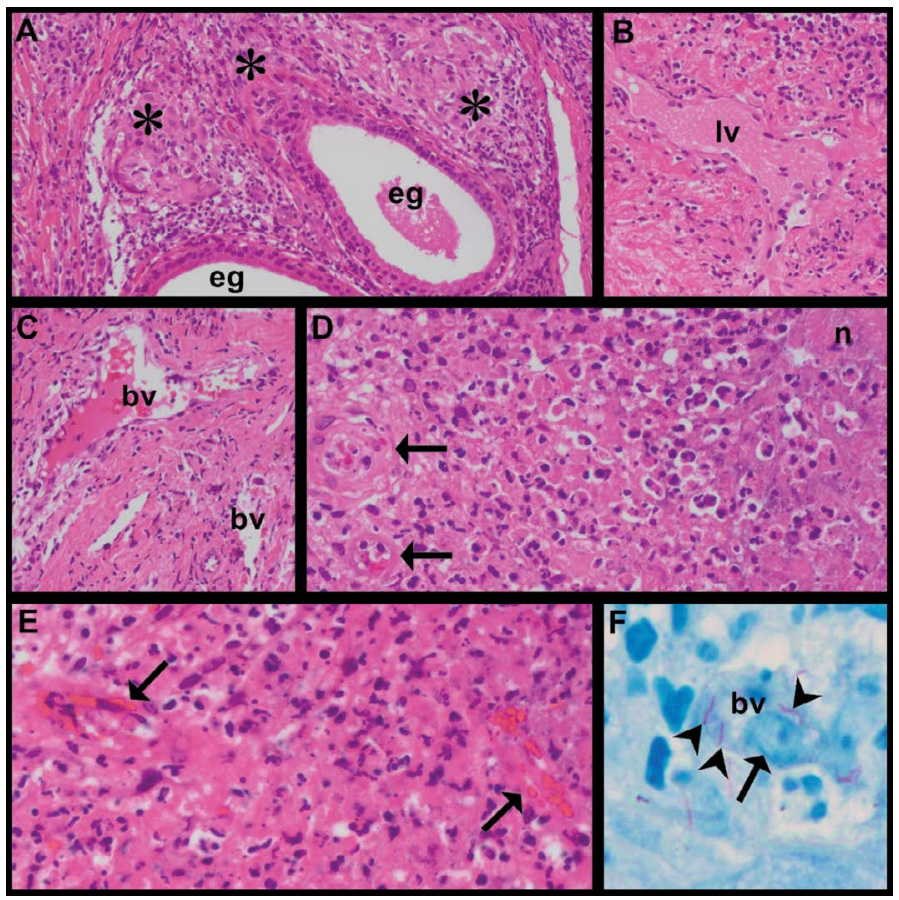

Figure 6: Microscopic features of clinical hypertrophic/edematous and abscessing lesions: Non-necrotizing granulomas (asterisks) around eccrine glands (eg) (A) (hematoxylin and eosin). Dilated lymphatic (B, Iv) and blood (C, bv) vessels (hematoxylin and eosin). Abscessing morphology (D) composed of sheets of neutrophils, cellular debris and necrosis ( $\mathrm{n}$ ) with vasculitis (arrows) and thrombophlebitis (E, arrows) (hematoxylin \& eosin). Acid fast bacilli in inflamed capillary (arrowheads) (bv=blood vessel, arrows=endothelial cells) (Ziehl Neelsen). 
the sentinel histomorphological classification of pulmonary TB. This classification assessed good, intermediate or poor histopathological host responses, with prioritized focus on the nature and extent of the necrosis and cellular responses, but did not incorporate organism density [25]. Approximately 12 years later, Ramanathan et al. [26] modified and adapted the Ridley and Ridley [25] classification for assessment of extrapulmonary nodal TB, the most common extrapulmonary TB location. Based mainly on the extent of granuloma organization or lack thereof, this group of workers classified nodal TB into microscopic hyperplastic, reactive, hyperactive and non-reactive microscopic forms. The organization of the granulomas referred to the usual/classical morphological architectural arrangement of central caseative necrosis and surrounding epithelioid histiocytes, giant cells, lymphocytes and plasma cells [26]. In this classification, the reactive type was the most common and the non-reactive type was the least common. More recently, Popescu et al. [2] modified the classification of Ramanathan et al. [26] to assess extrapulmonary, oral cavity TB by the presence or absence of Langhans giant cells in the hyperplastic granulomatous response. They also included the presence of suppurative necrosis and neutrophilic infiltration into the non-reactive/disorganized Stage IV response. Of 17 patients in this series [2], similar to that of nodal TB [26], the majority of oral cavity lesions were of reactive morphology.

The association between HIV infection/AIDS and TB, and atypical tuberculous manifestations, were noted within the first decade of recognition of AIDS as an emerging highly infectious and fatal disease [20,27]. Although the histomorphological range of HIVassociated TB (HIV-TB) has been documented rarely in pulmonary and extrapulmonary locations in autopsy, cytopathology and surgical pathology biopsy samples, the histomorphological range in humans remains poorly reported in all visceral locations $[19,28]$. Nambuya et al. [29] classified nodal HIV-TB into reactive $2+$, reactive $1+$, hyporeactive and non-reactive/anergic groups, based on decreasing granulomatous cellular characteristics and increasing acid fast bacillary numbers. Non-reactive TB is a histopathological entity, recognized in the preAIDS era, as foci of miliary necrosis containing large numbers of acid fast mycobacteria surrounded by a minimal tissue response or normal visceral tissue $[25,26]$. Because of the poor cellular response the concept of 'tuberculosis without tubercles' was coined a decade ago [28]. However, the non-reactive morphological tuberculous phenotype is not unique to AIDS, having been labeled "acute caseating TB", "Yersin type of TB" and "Landouzyxchekrankheit" in the pre-AIDS era [24]. To date, however, these classifications have not been employed to assess FGT TB in general or vulval TB, in particular, although the histomorphological descriptions of TB are relevant, especially in the HIV/AIDS context.

Considerable anatomic components of the vulva, including the mons pubis, vestibule, labium majus and labium minus, are cutaneous or mucocutaneous in nature. In the last 70 years, several classification systems of cutaneous TB, characterized by a plethora of clinicomorphological labels, histomorphologic diversity and varied MTb density have been created, but no single system has achieved universal acceptance by clinicians and pathologists [30-33]. The impact of HIV co-infection, AIDS and the synergistic immunosuppression on the cutaneous histomorphological profile has not been addressed in any cutaneous classification system, to date. Because of the exposed nature of the skin, the infectious profile of TB and HIV and the variability of referral patterns in health systems, not all patients with cutaneous $\mathrm{TB}$ are seen by dermatologists, or by specialists in referral centers, resulting in varied clinical descriptions in the global literature [34]. In the early 1900s the clinical morphology of vulval tuberculous lesions included mainly ulcerative or hypertrophic lesions [35]. In the last
40 years, additional descriptive terms have been employed, including nodules, ulcers, abscesses, papules, papulonecrotic discrete lesions or vulval hypertrophy and edema. To date, most of the described vulval histomorphology has focused on classic TB or tuberculous hypersensitivity responses [36-46].

Despite the common clinical descriptions in the present study, there was histomorphologic and bacillary density variability in lesions with similar clinical descriptions, viz. nodular, ulcerative, edematous/ hypertrophic and abscessing lesions. The histomorphological spectrum of the nodular clinical lesions included typical NGI with central caseative or suppurative inflammation and NNGI, as well as the less commonly documented pseudotumoral and anergic mononuclear inflammatory cell responses $[29,47]$. Whilst all suppurative granulomas were multibacillary, those with caseative necrosis were variably paucibacillary or multibacillary (Figures $1 \mathrm{~F}$ and $1 \mathrm{G}$ ). The latter was not a consistent reflection of a low CD4 count, as measured by the blood CD4 count. Further studies are necessary to determine whether the vulval cutaneous and mucocutaneous tissue has unique organ-specific immune factors that are not a reflection of circulating CD4 counts. It may be argued that granulomas with a neutrophilic response are multibacillary because the neutrophils are representative of earlier disease or that neutrophils are poorly phagocytic [22,29], but the hypothesis does not explain the varied bacillary load in established caseative granulomas. Three biopsies had, in addition, a plasma cellrich granulation tissue layer and NNGI that mimicked syphilitic vulval ulceration (Figure 3A). The absence of spirochetes and presence of acid fast bacilli (Figure 3B) favored TB. Ulceration was also noted microscopically in 4 lesions that had a nodular clinical appearance. In these biopsies, the ulcer bed lacked a zonation phenomenon and granulomas but contained granulation tissue with mixed inflammatory cells and myriad AFBs; the superficial, inflammatory granulation tissue response in 2 biopsies mimicked granuloma inguinale (Figure 3C). The diagnosis was based on the typical tuberculous NGI morphology of the deeper nodules that prompted Ziehl-Neelsen staining and mycobacterial identification, even in the granulation tissue (Figure 3D). Because of infective mimicry of TB by more common vulval infections, the importance of adequate biopsy size and depth in the histopathological diagnosis of vulval TB cannot be overemphasized.

Since the introduction of the entity 'mycobacterial pseudotumor' in 1992 [47], mycobacterial spindle cell pseudotumors have been reported in many extracutaneous sites including lung, lymph node, bone marrow, gastrointestinal tract and brain. To date, tuberculous spindle cell pseudotumoral lesions have not been diagnosed in the vulva. The present vulval pseudotumor mimicked carcinoma or lymphoma clinically. Heightened recognition of TB as a clinical tumor mimicker and increased suspicion of infective agents as causes of spindle cell pseudotumors microscopically, underpin the conduction of infective stains that are pivotal to optimal diagnosis. Infective spindle cell pseudotumors must be distinguished from HIV/AIDS-related spindle cell-rich tumors, such as Kaposi sarcoma, EBV-induced smooth muscle tumors and fibrous histiocytomas and non-AIDS defining spindle cell carcinomas and sarcomas [48].

One of the 9 nodular lesions (patient 1) had a non-specific, anergic chronic inflammatory histomorphology but was teeming with mycobacteria. In addition, two clinical abscessing lesions were characterized by an anergic, multibacillary abscessing phenotype, a poorly recognized histopathological entity [49]. This is a potentially serious diagnostic pitfall as abscesses of non-mycobacterial origin are common in all vulval mucocutaneous locations [50]. The anergic 
responses in pulmonary, nodal and oral cavity TB include non-specific, non-granulomatous, acute and chronic inflammatory responses [27-29]. At the histopathological diagnostic bench, a high index of suspicion and familiarity with the spectrum of anergic tuberculous morphology are the tocsins for Ziehl-Neelsen staining that underpin the confirmation of mycobacterial infection. Hence, a minimum good laboratory practice recommendation urges that all vulval abscesses should be subjected to Ziehl-Neelsen staining, especially in TB and/or HIV endemic regions.

The etiology of vulval ulcers is divergent and encompasses a spectrum of infective and non-infective causes [34]. While TB is not a common cause of vulval ulceration, ulceration is the most commonly reported clinical morphology of vulval TB [13-5]. In 1946, three morphological ulcer variants labeled 'lupus', 'scrofulodermoid' and 'noduloulcerative', were reported, with a common microscopic composition, including necrotizing granulomas and superficial nonspecific exudate [35]. In the present study, all biopsies from clinical ulcerative lesions had a microscopic zonation pattern (Figure 5A). Whilst such a zonation pattern has not been described in vulval tuberculous ulcers, to date, it is described in the walls of tuberculous pulmonary cavities and in papulonecrotic tuberculid [22,31,34,51]. In the present study, acid fast bacilli were identified in the zoned ulcerative inflammatory lesions in the vulva. While a biopsy of a papulonecrotic tuberculid was also described as ulcerative clinically in the present study, it lacked acid fast bacilli. This was congruent with the concept of tuberculids that, by definition, lack mycobacteria on histopathological appraisal and microbiological culture [34,51]. Tuberculids are cutaneous hypersensitivity tuberculous reactions in patients with visceral $\mathrm{TB}$, and include papulonecrotic tuberculid, lichen scrofulosorum, erythema induratum, nodular granulomatous phlebitis and nodular tuberculid $[31,33,34,52]$. Heightened recognition of vulval papulonecrotic tuberculid, a tuberculous granulomatous, ulcerative lesion with a zoned inflammatory pattern (Figure 5E) that lacks acid fast bacilli, is therefore necessary to avoid misdiagnosis of other infective, ulcerative granulomatous lesions, including syphilis, schistosomiasis and lymphogranuloma venereum [34].

Three patients in the present study had hypertrophic vulval lesions and vulval edema, a documented but under-recognized manifestation of vulval TB, attributed to the consequences of active chronic inflammation and lymphatic stasis [41]. Esthiomene, woody vulva, elephantiasis, cutaneous lymphangiectasia and lymphangioma circumscriptum have been recognized sequelae of such lymphatic obstruction, that may be associated with active inguinal tuberculous lymphadenitis $[10,36,38,41,53]$. Biopsies of 3 patients with clinically edematous vulval lesions and inguinal lymphadenopathy in the present study demonstrated the common finding of prominent dilated lymphatic and blood vessels (Figures $6 \mathrm{~B}$ and $6 \mathrm{C}$ ). The identification of active inguinal nodal TB on fine needle aspiration cytology in $2 / 3$ patients at the time of vulval biopsy streamlined the histopathological diagnosis of lichen scrofulosorum, that manifests microscopically as NNGI in cutaneous sub-epidermal, peri-appendigeal (Figure 6A) and perivascular locations. These vulval biopsies highlight the pivotal roles of the vulval biopsy for the recognition of the NNGI that has a wide etiological differential diagnosis, and obsessive clinicopathological correlation thereof, for the confirmation of lichen scrofulosorum. Vulval biopsy of the third patient revealed co-existent NNGI and NGI with acid fast bacilli. Lymphatic obstruction secondary to nodal TB is hypothesized as the cause of the vulval edema and hypertrophy in all 3 patients.
Infections may cause vasculitis by direct invasion of the wall of vessels of any size or by immune mechanisms, including immune complex disease and cryoglobulinemia [54,55,56]. Leucocytoclastic vasculitis may be caused by a range of viral, fungal, bacterial and parasitic diseases. While M.Tb and HIV may cause vascular pathology independently by several mechanisms, the presence of vasculitis in the context of TB and HIV co-infection is poorly reported [54-62]. The co-occurrence of TB and vasculitis was first reported in 1967 [55]. Circulating immune complexes, the levels of which are related to disease activity, have been demonstrated in pulmonary TB [55]. The 3 main presentations of cutaneous vasculitis, described in the context of pulmonary TB, are cutaneous leucocytoclastic vasculitis, Henoch Schonlein purpura and rifampicin-induced vasculitis [55]. Despite the heightened recognition of the spectrum of HIV-related infective vasculitic and vasculopathic reactions, the pathogenesis thereof remains enigmatic $[59,60]$. HIV-induced endothelial dysfunction, vascular wall injury, increased viral load and associated increase in inflammatory cytokines and tumour necrosis factor- $\alpha$ have been proposed $[59,60]$. Leucocytoclastic and granulomatous vasculitis, manifestations of type III and type IV hypersensitivity reactions [62], respectively, were present in 12 and 1 biopsy each, respectively, in the present study. Mycobacteria were identified in 5 biopsies within vessels that displayed leucocytoclastic vasculitis. Direct vascular wall injury by mycobacteria and a subsequent local type III immune complex-mediated hypersensitivity reaction is hypothesized in this context. Vasculitis is also a defining feature of selected tuberculids, viz. papulonecrotic tuberculid, erythema induratum, nodular tuberculid and nodular granulomatous phlebitis [34,52] that are associated with visceral TB. In the present study, the visceral tuberculous source for immune complex generation in the pathogenesis of the leucocytoclastic vasculitis in the papulonecrotic tuberculid was tuboovarian TB (Table 1).

In conclusion, this studyhighlights an expandinghistomorphological spectrum of vulval TB in the context of HIV co-infection and AIDS. Heightened awareness of this expanding spectrum is pivotal to optimal microscopic identification of TB in an anatomic location prone to a wide range of infective mimickers and will ensure timely treatment, not only of vulval TB, but also investigation of potential concomitant, active visceral $\mathrm{TB}$, follow-up thereof and holistic management of the afflicted patients. Lesions with a common clinical appearance had heterogeneous histomorphological features, while lesions with common histopathological features demonstrated divergent clinical morphology. Whether the anergic mononuclear or abscessing features, pseudotumoral spindle cell reaction, ulcers with a zoned inflammatory response and the presence of vasculitis of varied type are harbingers of the HIV-TB tissue partnership is debatable, but these features are not characteristic of classical TB histopathology and mandate further detailed investigation. Extrapulmonary TB does not share the global focus that pulmonary TB has received through the centuries, although it is associated with widespread anatomical morbidity. It is hoped that increased recognition of extrapulmonary FGT TB in general and vulval TB in particular, will encourage heightened clinicopathological reporting thereof, in an attempt to establish the spectrum of HIVassociated vulval $\mathrm{TB}$ and stimulate worldwide interest to investigate pathogenetic mechanisms thereof.

\section{Acknowledgement}

We thank Mrs M Moodley for assistance with manuscript formatting and upload, Mr Dinesh Sookhdeo for laboratory support and Mr S Bandezi, NHLS Management, for motivation and support of all research endeavours. 
Citation: Nhlonzi GB, Ramdial PK, Nargan K, Lumamba KD, Pillay B, et al. (2017) Vulval Tuberculosis: The Histomorphological Spectrum in Patients with HIV Co-Infection and AIDS. J AIDS Clin Res 8: 719. doi: 10.4172/2155-6113.1000719

Page 9 of 10

\section{References}

1. Montales MT, Chaudhury A, Beebe A, Patil S, Patil N (2015) HIV-associated TB syndemic: A growing clinical challenge worldwide. Front Public Health 3: 1-7.

2. Popescu MR, Pleşea IE, Olaru M, Strâmbu IR, Fronie Al, et al. (2015) Morphological aspects in tuberculosis of oral cavity-our experience and a review of the literature attempt. Rom J Morphol Embryol 56: 967-987.

3. Sandgren A, Hollo V, Van der Werf MJ (2013) Extrapulmonary tuberculosis in the European Union and European Economic Area, 2002 to 2011. Euro Surveill 18.

4. Kulchavenya E (2014) Urogenital tuberculosis: Definition and classification. Ther Adv Infect Dis 2: 117-122.

5. Arakeri SU, Sinkar P (2014) An unusual gross appearance of vulval tuberculosis masquerading as tumor. Case Rep Obstet Gynecol.

6. Mondal SK (2013) Histopathologic analysis of female genital tuberculosis: A fifteen year retrospective study of 110 cases in Eastern India. Turk Patoloji Derg 29: 41-45.

7. Botha MH, Van der Merwe FH (2008) Female genital tuberculosis. SA Fam Pract 50: 12-16.

8. Das P, Ahuja A, Gupta SD (2008) Incidence, etiopathogenesis and pathological aspects of genitourinary tuberculosis in India: A journey revisited. Indian J Urol 24: $356-361$

9. Dhall K, Das SS, Dey P (1995) Tuberculosis of Bartholin's gland. Int J Gynecol Obstet 48: 223-224.

10. Varma TR (2008) Tuberculosis of the female genital tract. Glob libr women's med.

11. Zajaczkowski T (2012) Genitourinary tuberculosis: historical and basic science review: Past and present. Cent Eur J Urol 65:182-187.

12. Duggal S, Duggal N, Hans C, Mahajan RK (2009) Female genital TB and HIV co-infection. Indian J Med Microbiol 27: 361-363.

13. Crossley J, Husain OAN (1958) Tuberculosis of the vulva. Br Med J 1: 1286.

14. Sardana K, Koranne RV, Sharma RC, Mahajan S (2001) Tuberculosis of the vulva masquerading as a sexually transmitted disease. J Dermatol 28: 505507

15. Wong S, Rizvi H, Cerio R, O'Toole EA (2010) An unusual case of vulval papulonecrotic tuberculid. Clin Exp Dermatol 36: 277-280.

16. Richter E, Schlüter C, Duchrow M, Hahn M, Rüsch-Gerdes S, et al. (1995) An improved method for the species-specific assessment of mycobacteria in routinely formalin-fixed and paraffin-embedded tissues. J Pathol 175: 85-92.

17. Li JYW, Lo STH, Ng C-S (2000) Molecular detection of Mycobacterium tuberculosis in tissues showing granulomatous inflammation without demonstrable acid-fast bacilli. Diagn Mol Pathol 9: 67-74.

18. World Health Organization (2016) Global tuberculosis report 2016. WHO.

19. Di Perri G, Cazzadori A, Vento S, Bonora S, Malena M, et al. (1996) Comparative histopathological study of pulmonary tuberculosis in human immunodeficiency virus-infected and non-infected patients. Tubercle Lung Dis 77: 244-249.

20. Korzeniewska-Kosela M, FitzGerald JM, Vedal S, Allen EA, Schechter MT, et al. (1992) Spectrum of tuberculosis in patients with HIV infection in British Columbia: Report of 40 cases. Can Med Assoc J 146: 1927-1934.

21. World Health Organisation (2015) Global Tuberculosis Report 2015. WHO.

22. Carnetti G (1955) The tubercle bacillus in the pulmonary lesion of man. Springer, New York.

23. Medlar EM (1955) The behavior of pulmonary tuberculous lesions. A pathological study. Amer Rev Tuberc 71: 1-244.

24. O’Brien JR (1954) Non-reactive tuberculosis. J Clin Pathol 7: 216-225.

25. Ridley DS, Ridley MJ (1987) Rationale for the histological spectrum of tuberculosis. A basis for classification. Pathology 19: 186-192.

26. Ramanathan VD, Jawahar MS, Paramasivan CN, Rajaram K, Chandrasekar $\mathrm{K}$, et al. (1999) A histological spectrum of host responses in tuberculous lymphadenitis. Indian J Med Res 109: 212-220.

27. Theuer CP (1989) Tuberculosis in patients with human immunodeficiency virus infection. Review of current concepts. West J Med 150: 700-704.
28. Horsburgh CR (1996) Tuberculosis without tubercles. Tubercle Lung Dis 77 197-198.

29. Nambuya A, Sewankambo N, Mugerwa J, Goodgame R, Lucas S (1988) Tuberculous lymphadenitis associated with human immunodeficiency virus (HIV) in Uganda. J Clin Pathol 41: 93-96.

30. Dias MFRG, Filho FB, Quaresma MV, do Nascimento LV, Da Costa Nery JA, et al. (2014) Update on cutaneous tuberculosis. An Bras Dermatol 89: 925-938.

31. Kumar B, Muralidhar S (1999) Cutaneous tuberculosis: A twenty year prospective study. Int J Tubercle Lung Dis 3: 494-500.

32. Sehgal VN, Wagh SA (1990) Cutaneous tuberculosis. Current Concepts. Int J Dermatol 29: 237-252.

33. Umapathy KC, Begum R, Ravichandran G, Rahman F, Paramasivan CN, et al. (2006) Comprehensive findings on clinical, bacteriological, histopathological and therapeutic aspects of cutaneous tuberculosis. Trop Med Int Health 11: 1521-1528.

34. Ramdial PK, Calonje E (2013) Vulvovaginal infections. Pathology of the vulva and vagina. Springer-Verlag, London 13-46.

35. Speiser MD, Guyer HB (11946) Tuberculous ulcer of the vulva. Am J Obstet Gynecol 51: 718-721.

36. Bhat RM, Saldanha CS, Kambil SM, Dandakeri S (2012) Cutaneous lymphangiectasia of the vulva secondary to tuberculosis. Indian J Sex Transm Dis 33: $35-37$.

37. Brenner BN (1976) Tuberculosis of the vulva. S Afr Med J 50: 1798-1800.

38. Goyal BK, Pradhan S, Jose T, Duggal BS, Sengupta P (2014) Woody Vulva: An unusual presentation of vulvar tuberculosis. J Obstet Gynecol India 64 S85-S87.

39. Jimenez-Gallo D, Navas-Garcia N, Albarran-Planelles C, Guerrero-Sanchez F (2012) Periorificial cutaneous tuberculosis of the vulva. Actas Dermosifiliogr 103: $929-930$

40. Millar JW, Holt S (1979) Vulval tuberculosis. Tubercle 60: 173-176.

41. Naik RPC, Srinivas CR, Balachandran C, Narayan PK, Ramnarayan K, et al. (1987) Esthiomene resulting from cutaneous tuberculosis of external genitalia Genitourin Med 63: 133-134.

42. Pandhi D, Mehta S, Singal A (2007) Genital tuberculid in a female child: A new entity (Childhood vulval tuberculid). Pediatr Dermatol 24: 573-575.

43. Shen HP, Chang WC, Hsieh CH, Yang TC, Hung YC (2011) Vulvar tuberculosis Tai J Obstet Gynecol 50: 106-108.

44. Stewart DB (1968) Ulcerative and hypertrophic lesions of the vulva. Proc Roy Soc Med 61: 363-365.

45. Talwar A, Puri N, Sandhu HPS (2009) Vulval lymphoedema following pulmonary tuberculosis. Int J STD AIDS 20: 437-439.

46. Varshney A, Goyal T (2011) Incidence of various clinic-morphological variants of cutaneous tuberculosis and HIV concurrence: a study from the Indian subcontinent. Ann Saudi Med 31: 134-139.

47. Logani S, Lucas DR, Cheng JD, loachim HL, Adsay NV (1999) Spindle cell tumors associated with mycobacteria in lymph nodes of HIV-positive patients: 'Kaposi sarcoma with mycobacteria' and 'mycobacterial pseudotumor. Am J Surg Pathol 23: 656-661.

48. Sing Y, Ramdial PK (2007) Cryptococcal inflammatory pseudotumors. Am J Surg Pathol 31: 1521-1527.

49. Berg J, Leonard A, Clancy CJ, Nguyen MH (1998) Subcutaneous abscesses due to mycobacterium tuberculosis: paradoxical expansion of disease during therapy for miliary tuberculosis. Clin Infect Dis 26: 231-232.

50. Lazenby GB, Thurman AR, Soper DE. Vulvar abscess.

51. Ramdial PK, Mosam A, Mallett R, Aboobaker J (1998) Papulonecrotic tuberculid in a 2-year-old girl: with emphasis on extent of disease and presence of leucocytoclastic vasculitis. Pediatr Dermatol 15: 450-455.

52. Hara K, Tsuzuki T, Takagi N, Shimokata K (1997) Nodular granulomatous phlebitis of the skin: A fourth type of tuberculid. Histopathology 30: 129-134.

53. Amouri M, Masmoudi A, Boudaya S, Amouri A, Ali IB, et al. (2007) Acquired lymphangioma circumscriptum of the vulva. Dermatol Online J 13: 10. 
Citation: Nhlonzi GB, Ramdial PK, Nargan K, Lumamba KD, Pillay B, et al. (2017) Vulval Tuberculosis: The Histomorphological Spectrum in Patients with HIV Co-Infection and AIDS. J AIDS Clin Res 8: 719. doi: 10.4172/2155-6113.1000719

54. Guillevin L (2013) Infections in vasculitis. Best Pract Res Clin Rheumatol 27: 19-31.

55. Parish WE, Rhodes EL (1967) Bacterial antigens and aggregated gamma globulin in the lesions of nodular vasculitis. Br J Dermatol 79: 131-147.

56. Teng GG, Chatham WW (2015) Vasculitis related to viral and other microbial agents. Best Pract Res Clin Rheumatol 29: 226-243.

57. Imanishi H, Tsuruta D, Oshima T, Sowa J, Mizuno N, et al. (2012) Cutaneous polyarteritis nodosa induced by Mycobacterium tuberculosis. J Dermatol 39: 738-739.

58. Mahmood FS, Schwatz E, Kurrup S, Sharp C, Hands G, et al. (2013) A diagnostic dilemma: differentiating between granulomatosis with polyangiitis and tuberculosis. Clin Med (Lond) 13: 411-413.

59. Patel N, Patel N, Khan T, Patel N, Espinoza LR (2011) HIV infection and clinica spectrum of associated vaculitides. Curr Rheumatol Rep 13: 506-512.

60. Pillay B, Ramdial PK, Naidoo DP (2015) HIV-associated large-vesse vasculopathy: A review of the current and emerging clinicopathological spectrum in vascular surgical practice. Cardiovasc J Afr 26: 70-81.

61. Singarayar J, Umerah B (1978) Tropical vasculitis and tuberculosis. Med J Zambia 12: 74-75.

62. Yao Y, Liu B, Wang JB, Li H, Liang HD (2010) Tuberculosis should not be ignored in patients with peripheral gangrene, J Vasc Surg 52: 1662-1664. 